\title{
Pharmacodynamics of Glyburide, Metformin, and Glyburide/Metformin Combination Therapy in the Treatment of Gestational Diabetes Mellitus
}

Diana L. Shuster ${ }^{1}$, Laura M. Shireman ${ }^{2}$, Xiaosu Ma ${ }^{3}$, Danny D. Shen ${ }^{2}$, Shannon K. Flood Nichols ${ }^{4}$, Mahmoud S. Ahmed ${ }^{5}$, Shannon Clark' ${ }^{5}$, Steve Caritis ${ }^{6}$ (D), Raman Venkataramanan ${ }^{6,7}$, David M. Haas ${ }^{8}$, Sara K. Quinney ${ }^{8}$ (D) , Laura S. Haneline ${ }^{9}$, Alan T. Tita ${ }^{10}$, Tracy A. Manuck ${ }^{11}$, Kenneth E. Thummel ${ }^{2}$, Linda Morris Brown ${ }^{12}$, Zhaoxia Ren ${ }^{13}$, Zane Brown ${ }^{14}$, Thomas R. Easterling ${ }^{14,15}$ and Mary F. Hebert ${ }^{14,15, *}$

In gestational diabetes mellitus (GDM), women are unable to compensate for the increased insulin resistance during pregnancy. Data are limited regarding the pharmacodynamic effects of metformin and glyburide during pregnancy. This study characterized insulin sensitivity (SI), $\beta$-cell responsivity, and disposition index (DI) in women with GDM utilizing a mixed-meal tolerance test (MMTT) before and during treatment with glyburide monotherapy (GLY, $n=38$ ), metformin monotherapy (MET, $n=34$ ), or GLY and MET combination therapy (COMBO; $n=36$ ). GLY significantly decreased dynamic $\beta$-cell responsivity (31\%). MET and COMBO significantly increased SI (121\% and 83\%, respectively). Whereas GLY, MET, and COMBO improved DI, metformin (MET and COMBO) demonstrated a larger increase in DI $(P=0.05)$ and a larger decrease in MMTT peak glucose concentrations $(P=0.03)$ than subjects taking only GLY. Maximizing SI with MET followed by increasing $\beta$-cell responsivity with GLY or supplementing with insulin might be a more optimal strategy for GDM management than monotherapy.

Study Highlights

\author{
WHAT IS THE CURRENT KNOWLEDGE ON THE \\ TOPIC? \\ $\square$ There are no data describing and comparing the mechanism \\ and magnitude of the clinical pharmacological effects of glybur- \\ ide (GLY), metformin (MET), and GLY and MET (COMBO) \\ therapy for gestational diabetes mellitus (GDM). \\ WHAT QUESTION DID THIS STUDY ADDRESS? \\ The objectives of this study were to characterize the pharma- \\ codynamic (PD) effects of GLY, MET, and COMBO therapy \\ for GDM management; evaluate the effects of gestational age \\ on insulin sensitivity (SI), $\beta$-cell responsivity, and disposition \\ index (DI); and evaluate the effects of genotype on MET PD \\ during pregnancy.
}

\begin{abstract}
WHAT DOES THIS STUDY ADD TO OUR KNOWLEDGE?

$\checkmark$ This is the first study to describe the PD of GLY, MET, and COMBO therapy in women with GDM as well as to report the effects of MATE2-K on MET's PD response (change in SI), OCT2 on MET dose, and PMAT on MET's effect on peak glucose concentrations during pregnancy.

HOW MIGHT THIS CHANGE CLINICAL PHARMACOLOGY OR TRANSLATIONAL SCIENCE?

Based on the clinical pharmacology gained in this study, maximizing MET and thereby SI first and then adding GLY to increase $\beta$-cell responsivity or adding insulin would be a more rational approach to GDM treatment. Further research is needed prior to making this a clinical recommendation.
\end{abstract}

\footnotetext{
${ }^{1}$ Clinical Pharmacology - Scientific Affairs, PRA Health Sciences, Lenexa, Kansas, USA; ${ }^{2}$ Departments of Pharmaceutics, University of Washington, Seattle, Washington, USA; ${ }^{3}$ Global PK/PD \& Pharmacometrics, Eli Lilly and Company, Indianapolis, Indiana, USA; ${ }^{4}$ Division of Maternal-Fetal Medicine, Department of Obstetrics and Gynecology, Madigan Army Medical Center, Tacoma, Washington, USA; ${ }^{5}$ Department of Obstetrics \& Gynecology, University of Texas Medical Branch in Galveston, Galveston, Texas, USA; ${ }^{6}$ Departments of Obstetrics \& Gynecology, University of Pittsburgh, Pittsburgh, Pennsylvania, USA; ${ }^{7}$ Department of Pharmacy, Pharmaceutical Sciences and Pathology, University of Pittsburgh, Pittsburgh, Pennsylvania, USA; ${ }^{8}$ Departments of Obstetrics \& Gynecology, Indiana University, Indianapolis, Indiana, USA; ${ }^{9}$ Department of Pediatrics, Indiana University, Indianapolis, Indiana, USA; ${ }^{10}$ Department of Obstetrics \& Gynecology, Center for Women's Reproductive Health, University of Alabama at Birmingham, Birmingham, Alabama, USA; ${ }^{11}$ Department of Obstetrics \& Gynecology, University of North Carolina, Chapel Hill, North Carolina, USA; ${ }^{12}$ RTI International, Environmental, and Health Science Unit, Biostatistics and Epidemiology Division, Rockville, Maryland, USA; ${ }^{13}$ Obstetric and Pediatric Pharmacology and Therapeutic Branch, Eunice Kennedy Shriver National Institute of Child Health and Human Development, Bethesda, Maryland, USA; ${ }^{14}$ Department of Obstetrics \& Gynecology, University of Washington, Seattle, Washington, USA; ${ }^{15}$ Department of Pharmacy, University of Washington, Seattle, Washington, USA. *Correspondence: Mary F. Hebert (mhebert@uw.edu)

Received June 20, 2019; accepted October 23, 2019. doi:10.1002/cpt.1749
} 
Insulin resistance and compensatory increase in insulin concentrations occur during normal pregnancy. Although insulin secretion does increase across gestation in women with gestational diabetes mellitus (GDM), there is an overall decreased ability to compensate for the degree of insulin resistance that occurs, leading to elevated blood glucose. ${ }^{1,2}$ If inadequately treated, GDM poses significant risks to the mother, fetus, and neonate. ${ }^{3,4}$ The American College of Obstetricians and Gynecologists (ACOG) recommends that all pregnant women be screened for GDM between 24 and 28 weeks gestation and on entry to prenatal care for those with risk factors. ${ }^{5}$

Treatments for women with GDM include dietary changes and pharmacotherapy. Historically, insulin has been the first-line therapy, but oral glucose-lowering drugs, such as metformin (MET) and glyburide (GLY) have increased in popularity because of their ease of administration, lower cost, and comparable efficacy to insulin. ${ }^{6,7}$ Recent GDM recommendations from $\mathrm{ACOG}^{4}$ exclude GLY from first-line oral treatment due to concerns regarding neonatal hypoglycemia and macrosomia. GLY, a second-generation sulfonylurea, increases insulin release. ${ }^{8} \mathrm{MET}$, an insulin sensitizer, lowers glucose concentrations by suppressing hepatic glucose production, decreasing glucose absorption, and increasing peripheral glucose uptake and utilization. ${ }^{9}$ A meta-analysis reported that MET was associated with more rapid glucose control and lower postprandial glucose concentrations, whereas GLY was associated with lower fasting glucose concentrations and hemoglobin A1Cs. ${ }^{10}$ Data are limited regarding the pharmacodynamics (PDs) of GLY and MET during pregnancy.

Biomathematical models, such as the oral minimal model of glucose and C-peptide kinetics, have been developed to describe the dynamic temporal relationship among serum glucose, insulin, and $\mathrm{C}$-peptide concentrations in response to the oral glucose tolerance test (OGTT) and the mixed meal tolerance test (MMTT). ${ }^{11-13}$ Insulin sensitivity (SI), $\beta$-cell responsivity, and disposition index (DI) have been utilized as quantitative indices to characterize the overall metabolic state in pregnant and nonpregnant individuals. ${ }^{11,14,15}$ Many factors contribute to glucose homeostasis; however, insulin secretion and action are the primary elements that determine response to glucose exposure and thereby glucose tolerance. The insulin secretory pathway is complex and regulated by multiple factors. Pancreatic $\beta$-cell function encompasses those processes leading to the release of insulin in response to elevated glucose concentrations. Exposure of $\beta$-cells to an abrupt increase of glucose elicits biphasic insulin secretion. The first phase involves the release of immediately available insulin-filled vesicles through exocytosis, the rate of which is defined as dynamic $\beta$-cell responsivity. It describes insulin secretion driven by the rate of change in glucose concentrations. The second phase involves the mobilization of reserved insulin granules to the cell membrane and their subsequent release, the rate of which is defined as static $\beta$-cell responsivity. It describes insulin secretion primarily driven by glucose concentrations. Total $\beta$-cell responsivity is controlled by both the static and dynamic components, as well as baseline $\beta$-cell responsivity, which represents the basal, nonstimulated index of insulin secretion. SI is a measure of the cascade of insulin actions needed to increase glucose utilization and suppress hepatic glucose production. Insulin resistance is a state of reduced SI, which can stem from defects in glucose transport as a result of impairments in translocation, fusion, exposure, or activation of GLUT-4 glucose transporters. DI describes the overall metabolic state and is a product of SI and total $\beta$-cell responsivity. It is an index of $\beta$-cell function, which takes into account the degree of insulin resistance. SI and total $\beta$-cell responsivity are hyperbolically related (i.e., reflecting the regulated feedback mechanisms that exist between blood glucose and insulin). ${ }^{16}$ These indices provide a mechanistic understanding of the patients' underlying disease pathology and response to pharmacotherapies.

Most oral GDM treatment strategies utilize either GLY or MET alone. However, the failure rates for individual agents are high (14-21\% for GLY and 40\% for MET). ${ }^{6,17-22}$ Considering the heterogeneous pathology of GDM (differing insulin resistance and/or $\beta$-cell dysfunction) and the different mechanisms of action for GLY and MET, some individuals might benefit from GLY or MET monotherapies and some might require GLY and MET combination (COMBO) therapy to optimize glycemic control. The objectives of this study were to (i) characterize the PD effects of GLY, MET, and COMBO in the management of GDM as determined by response to the oral MMTT; (ii) evaluate the effects of gestational age on SI, $\beta$-cell responsivity, and overall DI in healthy pregnant (HP) women; and (iii) evaluate the effects of genotype on MET PD response during pregnancy.

\section{MATERIALS AND METHODS \\ Subjects}

This was a multicenter, prospective, randomized, nonblinded phase I/ II longitudinal PD study (clinicaltrials.gov identifier NCT01329016). The study was approved by the institutional review boards at the University of Washington, Madigan Army Medical Center, University of Texas Medical Branch in Galveston, University of Pittsburgh, Indiana University, University of Utah Health Care, University of Alabama at Birmingham, and RTI International, and conducted in accordance with their guidelines. All subjects gave written, informed consent. There were two groups of women recruited for this study: pregnant women with a diagnosis of GDM $(n=109)$ and HP women $(n=30)$.

\section{Entry criteria}

GDM entry criteria included: pregnant women prior to 32 weeks gestation, singleton pregnancy, 18-45 years of age, failed diet therapy and required drug treatment. GDM diagnosis was made in one of three ways: (i) 3-hour OGTT (100 g glucose orally with two or more values meeting or exceeding targets: fasting $\geq 95 \mathrm{mg} / \mathrm{dL}, 1$-hour $\geq 180 \mathrm{mg} / \mathrm{dL}$, 2-hours $\geq 155 \mathrm{mg} /$ $\mathrm{dL}$ and 3-hours $\geq 140 \mathrm{mg} / \mathrm{dL}$ ), (ii) 2-hour OGTT (75 Gm glucose orally with one or more values meeting or exceeding targets: fasting $\geq 92 \mathrm{mg} / \mathrm{dL}$, 1-hour glucose $\geq 180 \mathrm{mg} / \mathrm{dL}$, 2-hour glucose $>153 \mathrm{mg} / \mathrm{dL}$ ), or (iii) 1-hour OGTT ( $50 \mathrm{Gm}$ glucose orally with 1 -hour glucose $\geq 185$ ). Exclusion criteria for women with GDM included: medications expected to interact with GLY or MET, medications expected to alter blood glucose concentrations, serum creatinine $>1.2 \mathrm{mg} / \mathrm{dL}$; hematocrit $<28 \%$; allergy to glyburide, metformin, or sulfa-drugs; significant liver disease; congestive heart failure or history of myocardial infarction; moderate to severe pulmonary disease; and adrenal or pituitary insufficiency. Healthy pregnant women entry criteria included: singleton pregnancy, 18-45 years of age, between 20 and 32 weeks gestation, and a normal 1-hour or 2 -hour OGTT. Exclusion criteria for HP women included: hematocrit $<28 \%$ or known kidney, liver, heart, pulmonary, adrenal, or pituitary disease as well as drugs that alter glucose concentrations. 


\section{Diagnosis and treatment}

Subjects with GDM were randomized to GLY, MET, or COMBO, and initial dosage and escalation were determined per treatment algorithms as seen in Supplemental Figures S2-S4. Provider discretion was allowed. Blood glucose concentrations were considered controlled when $\geq 75 \%$ of fasting glucose concentrations were $\leq 95 \mathrm{mg} / \mathrm{dL}$ and $\geq 75 \%$ of either 1-hour postprandial glucose concentrations were $<140 \mathrm{mg} /$ $\mathrm{dL}$ or 2-hour postprandial glucose concentrations were $<120 \mathrm{mg} / \mathrm{dL}$. Glyburide initial dosage was $2.5 \mathrm{mg}$ orally twice daily. Doses were titrated until glucose concentrations were considered controlled with maximum dosage of $8.75 \mathrm{mg}$ orally 3 times daily. MET initial dosage was $500 \mathrm{mg}$ twice daily and titrated to clinical control. In the COMBO group, subjects received $2.5 \mathrm{mg}$ of GLY and $500 \mathrm{mg}$ of MET twice daily initially and titrated to clinical control. If subjects did not achieve glycemic control by titration of dosage according to their treatment algorithm, then subjects completed study day 2 (SD2) and medications were switched or adjusted per provider's preference. Treatment was initiated at $\leq 32$ weeks of gestation. GLY and MET administrations were not controlled for fasting or fed conditions except on SD2. On SD2, GLY and/or MET were administered simultaneously with initiation of the MMTT. Subjects were determined to be nonadherent if they did not adhere to their treatment regimen based on study pill count or physician clinical impression or did not follow study protocol.

\section{MMTT}

PD parameters were estimated prior to study day 1 (SD1) and during treatment (SD2) utilizing an MMTT consisting of one can of Boost Plus energy drink, two slices of whole wheat toast, and two teaspoons of margarine, which was consumed within 10 minutes. SD2 took place once subjects achieved clinical control or prior to switching therapy if they failed to achieve glycemic control. Serial blood samples were collected pre-MMTT (time $=0$ ), and 10, 20, 30, 60, 90, 120, 150, 180, 210, and 240 minutes following the initiation of the MMTT to measure serum glucose, insulin, and $\mathrm{C}$-peptide concentrations. Glucose concentrations were measured using a glucose oxidase/peroxidase assay. ${ }^{23}$ Insulin and C-peptide concentrations were measured using previously described radioimmunoassays. ${ }^{24,25}$

\section{MMTT parameter estimation}

Insulin sensitivity, $\beta$-cell responsivity, and DI were estimated as previously described. ${ }^{11-13,15,26-28}$ Model parameters were estimated for individual subjects by nonlinear least squares regression using the SAAM II software version 2.3 (The Epsilon Group, Charlottesville, VA). Area under the curves (AUCs) for glucose, C-peptide, and insulin were calculated utilizing trapezoidal rule in $\mathrm{R}^{29} \mathrm{PD}$ response was defined as an increase in PD parameter estimates on SD2 relative to SD1 based on the known mechanisms of action for the drug (i.e., GLY increases total $\beta$-cell responsivity, MET increases SI, and COMBO therapy increases either or both parameters). Gestational age-matched HP subjects were included in this study to estimate and correct for gestational age-dependent changes in PD parameters between SD1 and SD2. The correction for gestational age-dependent effects was accomplished by subtracting the average difference between SD2 and SD1 in the HP subjects from individual GDM subjects' SD2 parameters.

\section{Genotyping}

DNA was isolated from whole blood, and genotypes were determined using validated TaqMan assays. Maternal samples were assayed for OCT1: SLC22A1 (rs622342); OCT2: SLC22A2c.808G>T polymorphism (rs316019); MATE1: SLC47A1 (rs2289668), and (rs8065082); MATE2-K: -130G>A polymorphism (rs12943590); as well as PMAT (rs2685753) and (rs6971788).

\section{Statistical analyses}

Differences in PD parameter estimates between SD1 and SD2 were estimated using a paired Student's $t$-test or between arms of the study using an unpaired Student's $t$-test or analysis of variance. A $\chi^{2}$ test was used to compare race and ethnicity between study arms. Results are reported as mean \pm SD (95\% confidence interval). No adjustments for multiple testing were performed. All statistical analyses and graphs were done in R. ${ }^{29,30}$

\section{RESULTS \\ Demographics}

Demographics for adherent subjects who completed the study are reported in Table 1. Notably, healthy pregnant women were significantly younger and weighed less than those with GDM. Demographics for all subjects can be found in Supplemental Table S1. Nineteen subjects with GDM completed SD1, but not SD2. Reasons for withdrawal included: three were lost to follow-up, seven went into early delivery, one had scheduling difficulties, one had medication nonadherence, one became ineligible, three started alternate therapy, one experienced anxiety with blood draws, one transferred care, and one is unknown. Results are reported for adherent subjects who completed the study.

\section{Glucose, insulin, and C-peptide concentrations}

Average serum concentration-time profiles for glucose, insulin, and C-peptide during a 4-hour MMTT on SD1 and SD2 (during treatment for subjects with GDM) are shown in Figure 1 for GDM and HP groups. Mean glucose AUCs were lower on SD2 in the COMBO $(P<0.001)$ and $\operatorname{MET}(P=0.004)$ groups, and not significantly different in the GLY $(P=0.5)$ and HP $(P=0.8)$ groups. Mean insulin AUCs were lower for the MET group on SD2 than SD1 $(P=0.02)$ but not significantly different in any other groups. Mean C-peptide AUCs were similar before and with treatment in the COMBO $(P=0.3)$ and MET groups $(P=0.2)$. However, GLY and HP groups had higher C-peptide AUCs on SD2 than SD1 (GLY $P=0.01$; HP $P<0.001$ ).

\section{Gestational age-dependent changes}

The hyperbolic relationship between total $\beta$-cell responsivity and SI in HP women is shown in Figure 2. From SD1 (30 \pm 1 weeks gestation) to SD2 (36 \pm 1 weeks gestation), the hyperbolic mean DI curve shifted down and to the left. Baseline $\beta$-cell responsivity increased by an average of $31 \%(P<0.001)$ and overall DI decreased by $6 \%(P=0.04)$ between SD1 and SD2 (Table 2$)$. Other parameters were not significantly different between study days.

\section{PD parameters}

All SD2 PD parameters for subjects with GDM are adjusted for gestational age. The mean hyperbolic relationships between total $\beta$-cell responsivity and SI for HP women and women with GDM on SD1 are depicted in Figure 3. The vectors on the graph depict the mean PD effects of GLY, MET, and COMBO therapy in subjects with GDM. MMTT PD parameters for subjects with GDM are reported in Table 3.

In the GLY group, dynamic $\beta$-cell responsivity decreased by an average of $31 \%(P<0.001)$, whereas baseline $\beta$-cell responsivity increased by $62 \%(P=0.03)$ and DI $119 \%(P=0.04)$. No significant effects were seen in other PD parameters. In the MET group, SI increased by $121 \%(P=0.005)$; DI $203 \%$ $(P=0.003)$, total $\beta$-cell responsivity $31 \%(P=0.04)$, and static 
Table 1 Demographics and study drug dosing for adherent subjects with gestational diabetes and gestational age-matched HP subjects who completed the study

\begin{tabular}{|c|c|c|c|c|c|}
\hline & All GDM & сомво & GLY & MET & HP \\
\hline$n$ & 89 & 32 & 32 & 25 & 28 \\
\hline \multicolumn{6}{|l|}{ Race/ethnicity } \\
\hline White, \% & & 81.2 & 78.1 & 80.0 & 82.1 \\
\hline Black, \% & & 9.4 & 21.9 & 16.0 & 17.9 \\
\hline Hispanic, \% & & 34.4 & 40.6 & 36.0 & 32.1 \\
\hline Asian, \% & & 5.6 & 0 & 4.0 & 0 \\
\hline Native American, \% & & 2.8 & 0 & 0 & 0 \\
\hline Age SD1, years & $30 \pm 5(19-42)$ & $29 \pm 4(19-37)$ & $30 \pm 6(20-42)$ & $31 \pm 4(22-39)$ & $\begin{array}{l}25 \pm 5(18-38) \\
(P<0.001)^{a}\end{array}$ \\
\hline Height SD1, cm & $162 \pm 6(147-179)$ & $162 \pm 7(153-175)$ & $161 \pm 6(147-173)$ & $162 \pm 6(147-179)$ & $162 \pm 8(147-178)$ \\
\hline Body weight SD1, kg & $90 \pm 20(60-200)$ & $100 \pm 20(60-200)$ & $90 \pm 20(60-200)$ & $90 \pm 10(70-100)$ & $\begin{array}{c}80 \pm 10(50-100) \\
(P<0.001)^{\mathrm{a}}\end{array}$ \\
\hline BMI Pre-pregnancy, kg/m² & $33 \pm 7(20-55)$ & $34 \pm 8(22-55)$ & $33 \pm 6(20-53)$ & $31 \pm 6(21-43)$ & $\begin{array}{c}27 \pm 5(20-40) \\
(P<0.001)^{\mathrm{a}}\end{array}$ \\
\hline GA, SD1, weeks & $30 \pm 2(19-33)$ & $30 \pm 3(19-33)$ & $30 \pm 2(24-33)$ & $31 \pm 2(20-33)$ & $30 \pm 1(28-33)$ \\
\hline GA, SD2, weeks & $35 \pm 2(26-39)$ & $35 \pm 3(26-38)$ & $36 \pm 2(32-39)$ & $35 \pm 1(32-38)$ & $36 \pm 1(34-38)$ \\
\hline $\begin{array}{l}\text { Glyburide dose SD2, } \\
\text { mg/day }\end{array}$ & $8 \pm 6(1-30)$ & $6 \pm 4(1-20)$ & $10 \pm 6(2-30)$ & NA & NA \\
\hline $\begin{array}{l}\text { Metformin dose SD2, } \\
\text { mg/day }\end{array}$ & $\begin{array}{c}1,300 \pm 500 \\
(1,000-2,600)\end{array}$ & $\begin{array}{c}1,200 \pm 500 \\
(1,000-2,600)\end{array}$ & NA & $\begin{array}{c}1,400 \pm 500 \\
(1,000-2,000)\end{array}$ & NA \\
\hline
\end{tabular}

$\mathrm{BMI}$, body mass index; COMBO, glyburide plus metformin combination therapy; GA, gestational age; GDM, gestational diabetes mellitus; GLY, glyburide monotherapy; HP, healthy pregnant subjects; MET, metformin monotherapy; SD1, study day 1; SD2, study day 2.

${ }^{\text {aS }}$ ignificantly different than all GDM subjects, results reported as mean \pm SD (range).
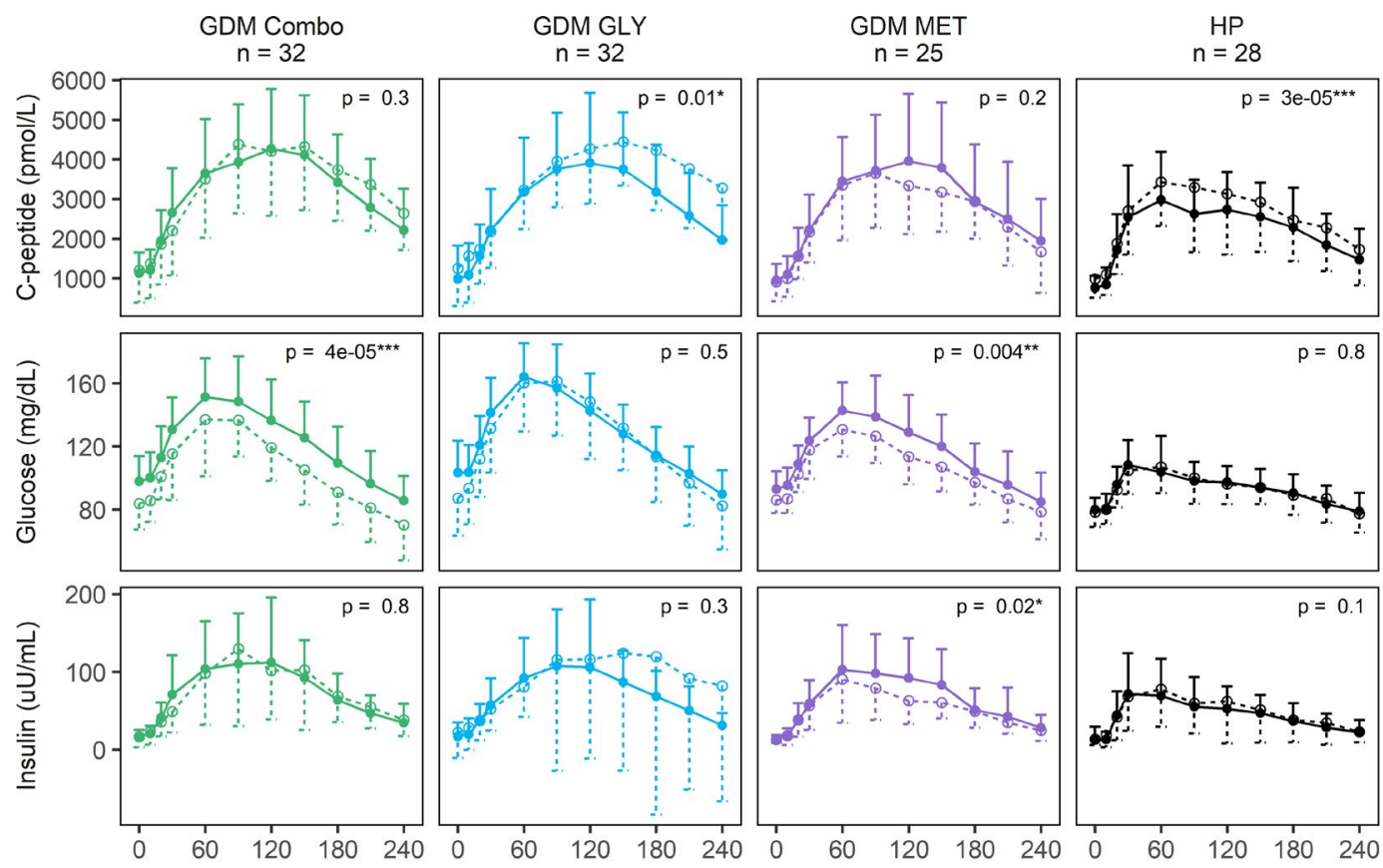

Arm
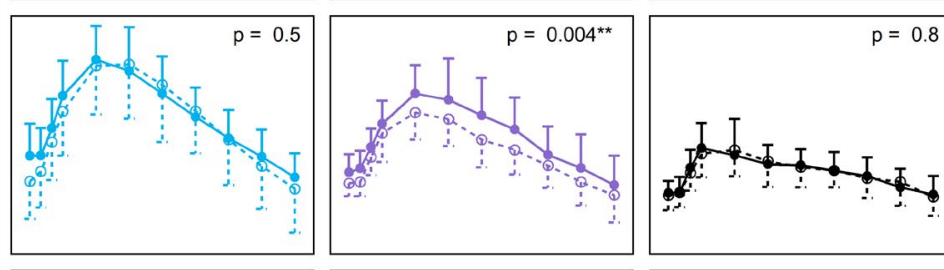

$\rightarrow$ GDM Combo

$\rightarrow$ GDM GLY

$\rightarrow$ GDM MET

$\rightarrow$ HP
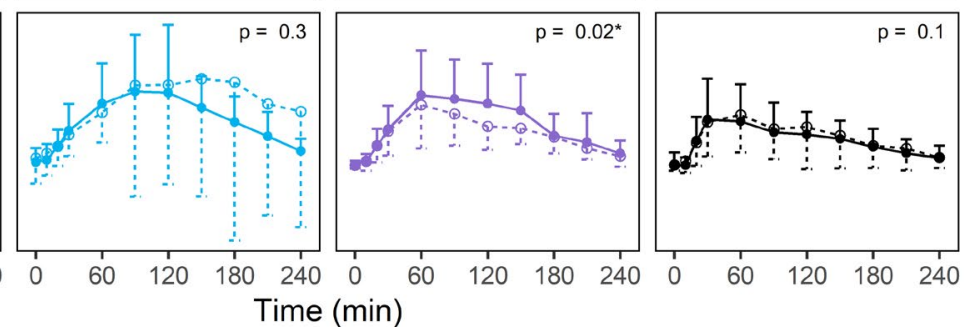

Study Day

$\rightarrow 1$

$-0-2$

Figure 1 Glucose, insulin, and C-peptide concentration-time profiles for all subjects with gestational diabetes mellitus (GDM) and healthy pregnant (HP) subjects who completed the study and were adherent to study procedures. Shown are mean concentrations at each time point with the SD represented by unidirectional error bars. Time since initiation of the mixed-meal tolerance test and dose in minutes is listed on the $x$ axis and the concentration of C-peptide (pM), glucose $(\mathrm{mg} / \mathrm{dL})$, or insulin $(\mu \mathrm{U} / \mathrm{mL})$ is on the $y$ axis. Data for study day 1 are shown with filled circles (means), solid lines, and upper error bars (SD); data for study day 2 are shown with open circles, dashed lines, and lower error bars. Treatment arms are represented by $\mathrm{COMBO}=$ metformin/glyburide combination therapy group (green), MET = metformin monotherapy group (purple), and GLY = glyburide monotherapy group (blue), and HP group (black). Significance is indicated by asterisks. 


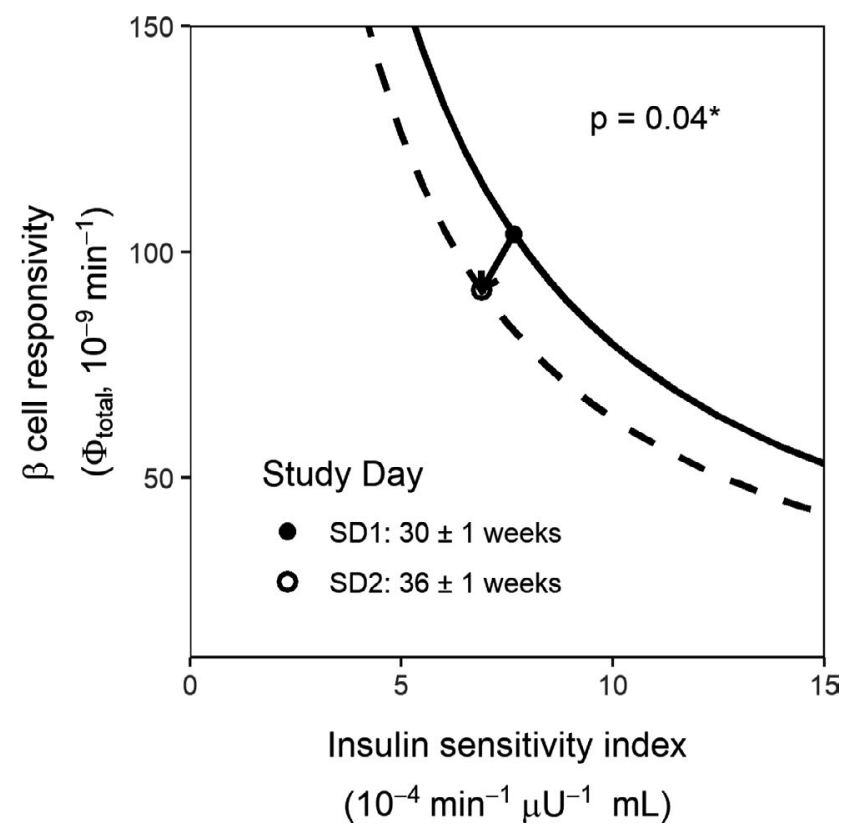

Figure 2 Effect of gestational age on the mean disposition index in healthy pregnant (HP) subjects. Only paired data for HP subjects who completed study day 1 (SD1) and study day 2 (SD2) are included $(n=28)$. Insulin sensitivity $(\mathrm{SI})$ is depicted on the $x$ axis, and total $\beta$ cell responsivity is depicted on the $y$, and points depict the mean total $\beta$-cell responsivity and mean SI on SD1 (filled circle) and SD2 (open circle) for the HP group. The hyperbolas shown depict the calculated total $\beta$-cell responsivity given the range of SI values shown for HP on SD1 (solid line) and SD2 (dashed line) where total $\beta$-cell responsivity $=\frac{\mathrm{DI}}{\mathrm{SI}}$ and the $\mathrm{DI}$ value used was the mean total $\beta$-cell responsivity times the mean SI.

$\beta$-cell responsivity $33 \%(P=0.04)$, whereas baseline $\beta$-cell responsivity decreased $28 \%(P=0.004)$, and MMTT peak glucose concentration $7 \%(P=0.006)$. There was no significant effect on dynamic $\beta$-cell responsivity. In the COMBO group, SI increased by $83 \%(P=0.03)$, total $\beta$-cell responsivity $57 \%$
$(P=0.004)$, static $\beta$-cell responsivity $72 \%(P=0.002)$, and DI 224\% $(P<0.001)$. No significant effects were seen in other PD parameters. The change in DI for all GDM subjects taking MET, combining those in the MET and COMBO groups, was greater than for the GLY group $(P=0.05)$.

Distributions for changes in PD parameters are depicted in Supplemental Figure S1. In the subjects with GDM, 56\% of subjects in the GLY and $74 \%$ of subjects in the COMBO group exhibited some pharmacologic response to GLY (increase in total $\beta$-cell responsivity). In addition, $84 \%$ of subjects in the MET group and $74 \%$ of subjects in the COMBO group exhibited pharmacologic response to metformin (increase in SI). In the COMBO group, pharmacologic response to either GLY and/or MET (increase in total $\beta$-cell responsivity and/or SI) was seen in $90 \%$ of subjects and pharmacologic response to both GLY and MET (increase in total $\beta$-cell responsivity and SI) was seen in $58 \%$.

\section{Dosage}

Table 1 includes average GLY and MET dose/day on SD2 for subjects with GDM in the COMBO, GLY, and MET groups. Mean GLY dose/day was higher in the GLY group than in the COMBO group $(P=0.005)$. The mean MET dose/day was numerically higher in the MET group than in the $\mathrm{COMBO}$ group but failed to achieve significance $(P=0.1)$.

\section{Effect of genotype on MET PD response and MET dose}

Figure 4a-c depicts the association among MET transportersMATE2-K, PMAT, and OCT2 genotypes and MET response or dose.

\section{MATE2-K and metformin's effect on SI}

Women with GDM with MATE2-K (rs12943590; G>A) AA genotype had a smaller change in SI with MET than those with MATE2-K GG genotype (Figure 4a; $P=0.03$ ).

Table 2 Mixed meal tolerance test parameters for HP control subjects SD1 (baseline) and SD2

\begin{tabular}{|c|c|c|c|}
\hline \multirow[b]{2}{*}{ Parameter } & \multicolumn{3}{|c|}{$\mathrm{HP}(n=28)$} \\
\hline & SD1 & SD2 & $\Delta$ \\
\hline $\mathrm{SI}\left(10^{-4}\right.$ minute $\left.^{-1} \mu \mathrm{U}^{-1} \mathrm{~mL}\right)$ & $8 \pm 6(2-20)$ & $7 \pm 4(1-20)$ & $\begin{array}{c}-0.8 \pm 4(-9 \text { to } 6) \\
(P=0.6)\end{array}$ \\
\hline$\Phi_{\text {total }}\left(10^{-9}\right.$ minute $\left.^{-1}\right)$ & $120 \pm 60(51-280)$ & $110 \pm 40(46-190)$ & $\begin{array}{c}-10 \pm 50(-100 \text { to } 20) \\
(P=0.1)\end{array}$ \\
\hline$\Phi_{\text {static }}\left(10^{-9}\right.$ minute $\left.^{-1}\right)$ & $100 \pm 50(30-200)$ & $80 \pm 30(30-200)$ & $\begin{array}{c}-10 \pm 40(-100 \text { to } 30) \\
(P=0.1)\end{array}$ \\
\hline$\Phi_{\text {dynamic }}\left(10^{-9}\right)$ & $\begin{array}{l}3,000 \pm 2,000 \\
(1,000-7,000)\end{array}$ & $3,000 \pm 1,000(1,000-5,000)$ & $\begin{array}{c}2 \pm 1,000(-2,000 \text { to } 2,000) \\
(P=1)\end{array}$ \\
\hline$\Phi_{\text {baseline }}\left(10^{-9}\right.$ minute $\left.^{-1}\right)$ & $10 \pm 4(4.3-20)$ & $14 \pm 6(6.2-26)$ & $\begin{array}{c}3 \pm 3(-0.2 \text { to } 9) \\
(P<0.001)\end{array}$ \\
\hline $\mathrm{DI}\left(10^{-13}\right.$ minute $\left.^{-2} \mu \mathrm{U}^{-1} \mathrm{~mL}\right)$ & $800 \pm 500(200-2,000)$ & $600 \pm 300(200-1,000)$ & $\begin{array}{c}-200 \pm 400(-1,000 \text { to } 400) \\
(P=0.04)\end{array}$ \\
\hline Peak glucose (mg/dL) & $120 \pm 20(94-150)$ & $120 \pm 10(96-150)$ & $\begin{array}{c}-0.7 \pm 10(-30 \text { to } 10) \\
(P=0.8)\end{array}$ \\
\hline
\end{tabular}

Results reported as mean \pm SD (95\% confidence interval).

$\Delta$, average change between study day 1 and study day $2 ; \Phi, \beta$-cell responsivity; DI, disposition index; SD1, study day 1 ; SD2, study day 2 ; SI, insulin sensitivity. 


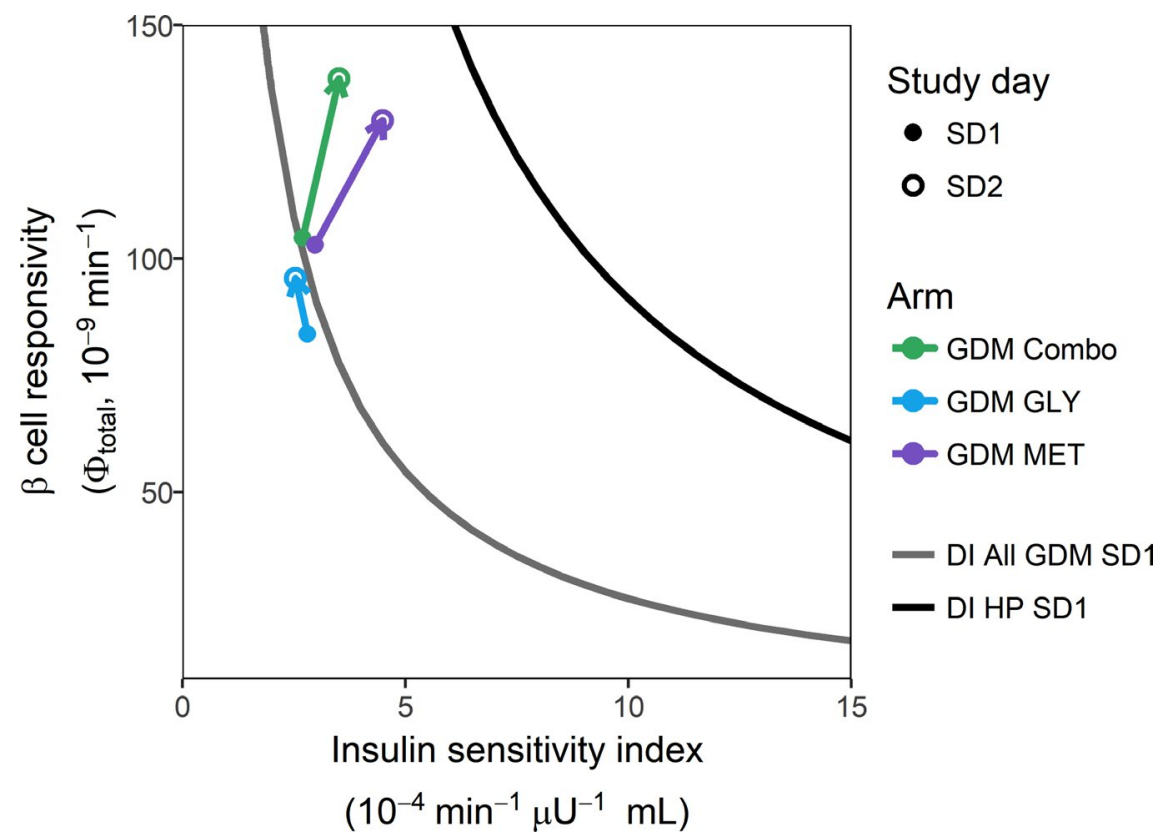

Figure 3 Pharmacodynamic effects of glyburide (GLY), metformin (MET), and GLY/MET combination (COMBO) therapies. Gray line depicts the mean baseline disposition index for all adherent subjects with gestational diabetes mellitus (GDM) who completed the study. The black line depicts the mean baseline disposition index for all healthy pregnant (HP) subjects who completed the study. The vectors depict the mean pharmacodynamic effect of GLY (blue arrow), MET (purple arrow), and GLY/MET COMBO therapy (green arrow). Solid dots represent mean baseline disposition index study day 1 (SD1) and open circles represent study day 2 (SD2) mean disposition index adjusted for mean gestational age-dependent change.

\section{PMAT and MET effect on peak glucose concentration} PMAT (rs2685753 G>A) AG genotype was associated with a larger decrease in MMTT peak glucose concentrations than GG genotype (Figure $\mathbf{4 b} ; P=0.03$ ).

\section{OCT2 effect on MET dose}

Women with OCT2 (rs316019) AC genotype, on average, required a higher MET dose than those with OCT2 CC genotype (Figure 4c; $P=0.001$ ). There were no subjects with AA genotype.

In this study, MATE1, PMAT, OCT1, and OCT2 genotypes were not associated with MET pharmacologic effect (change in SI); MATE1, MATE2-K, PMAT, and OCT1 genotypes were not found to significantly affect MET dose; and MATE1, MATE2-K, OCT1, and OCT2 genotypes did not alter metformin's effect on MMTT peak glucose concentrations.

\section{DISCUSSION}

The majority of medication prescribing during pregnancy is based on clinical trials in the nonpregnant population. The mechanism and magnitude of pharmacologic response to GLY and MET have not been described during pregnancy despite the significant alterations in glucose and insulin handling that occur during normal pregnancy. This study is the first to (i) quantify and compare the PD effects of GLY, MET, and COMBO treatment in pregnant women with GDM, and (ii) report the effects of MATE2-K on metformin's PD response (change in SI), OCT2 on MET dose, and PMAT on MET's effect on peak glucose concentrations during pregnancy.
Most healthcare providers who prescribe oral glucose-lowering agents for women with GDM currently use either MET or GLY monotherapies, with significant failure rates. ${ }^{22}$. This may occur for two reasons. First, evidence suggests GLY and MET exposures are reduced during pregnancy ${ }^{11,31}$; therefore, utilizing dosage strategies established in nonpregnant individuals might not be appropriate. For this reason, GLY dosage up to $8.75 \mathrm{mg}$ orally 3 times daily was allowed in this study. Second, the underlying pathology of GDM is heterogeneous with respect to $\beta$-cell dysfunction and insulin resistance. Monotherapies that treat only one facet of GDM pathology may not be as effective as a combination approach. The vectors depicted in Figure 3 demonstrated the average response to each approach. As expected, GLY (average dose: $10 \mathrm{mg} /$ day) exhibited its effects primarily through $\beta$-cell responsivity and produced a small average DI vector. Although MET (average dose: 1,400 mg/day) improved SI as expected, it also improved $\beta$-cell responsivity. The addition of GLY (average dose: $6 \mathrm{mg} /$ day) to MET (average dose: $1,200 \mathrm{mg} /$ day) in the COMBO group boosted the average effect on $\beta$-cell responsivity, but had less effect on SI than the MET group, as the dose of MET in the COMBO group was, on average, $200 \mathrm{mg} /$ day lower. Interestingly, none of the drug treatment vectors moved the average DI back to what would be considered normal in pregnancy. Given the hyperbolic nature of the DI curve and where the women with GDM started at baseline, the treatments that improved SI most moved the subjects closer to a normal DI than those that primarily affected $\beta$-cell responsivity (i.e., the change in DI for women with GDM taking MET in the MET and COMBO groups was greater than in those taking GLY). The majority of women with GDM initiated treatment with a DI on the steep ascending portion 


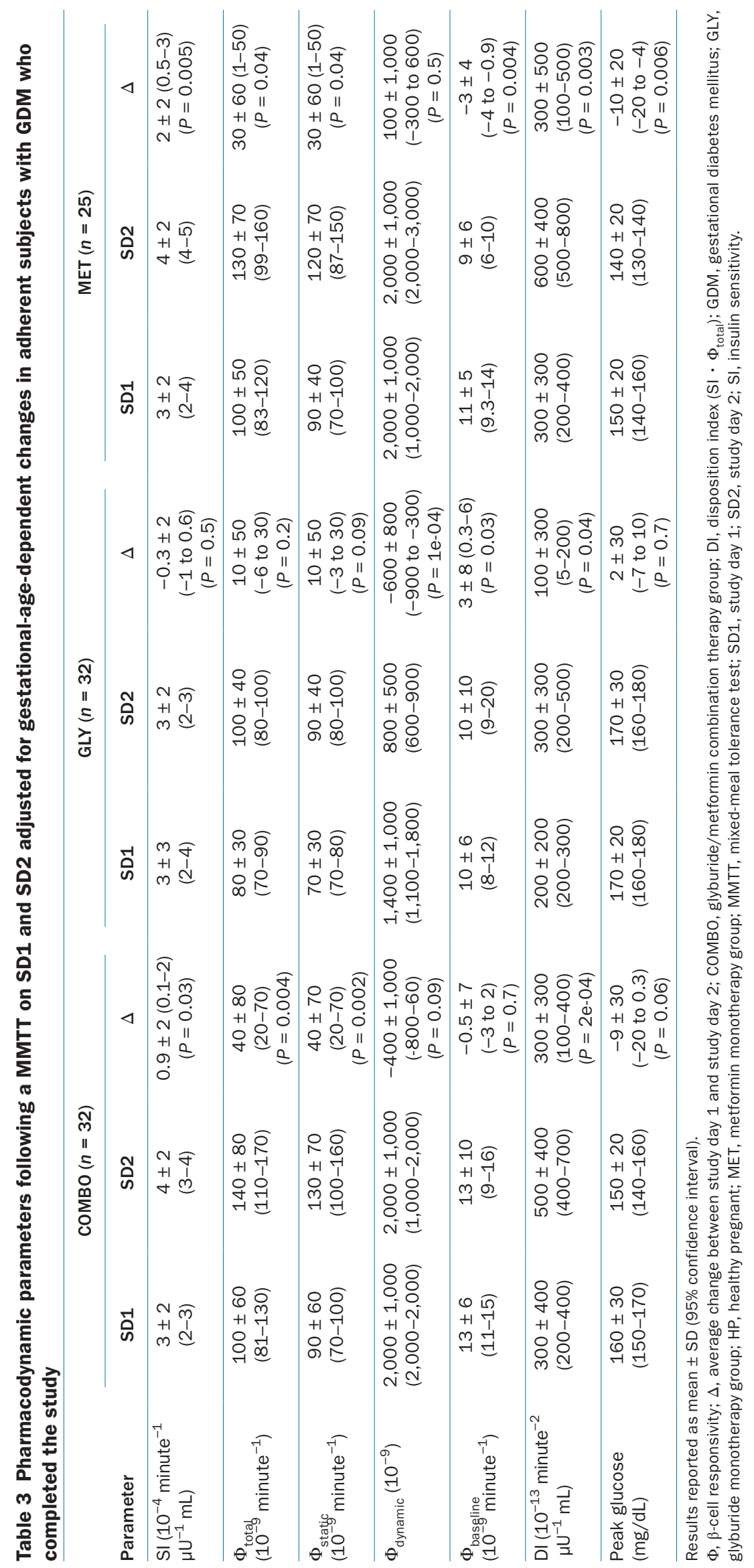


of the hyperbolic DI curve, making GLY (i.e., altering $\beta$-cell responsivity alone) far less successful in normalizing overall DI. This suggests that maximizing MET and thereby SI first and then adding GLY to increase $\beta$-cell responsivity or adding insulin supplementation would be a more rational approach to managing patients with GDM. Further research is needed prior to making this a clinical recommendation. However, this is consistent with recent ACOG recommendations for women who decline insulin to use MET as the first-line alternative oral agent. ${ }^{4}$

In this study, the percent of subjects having some pharmacologic response (i.e., improvement in either SI and/or total $\beta$-cell responsivity) differed by treatment arm. Fifty-six percent of the GLY group, $84 \%$ of the MET group, and $90 \%$ of the COMBO group had some pharmacologic response to GLY and/or MET. Both $\mathrm{COMBO}$ and MET were associated with significant reductions in MMTT average glucose exposure, whereas GLY, on average, was not (Figure 1). The Hyperglycemia and Adverse Pregnancy Outcomes (HAPO) study found a strong association between higher maternal glucose concentrations in response to an OGTT and higher risk of pregnancy complications from GDM. ${ }^{32}$ This supports the merit of clinical trials exploring comparative efficacy of MET either alone or in combination.

Consistent with the expected effect of GLY, average MMTT C-peptide concentrations were higher in the GLY group with treatment than before treatment (Figure 1). Although insulin concentrations were numerically higher with GLY, this did not reach statistical significance. Interestingly, in the COMBO group, neither average $\mathrm{C}$-peptide nor insulin concentrations were significantly higher, perhaps due to the lower GLY dosage in the COMBO group than in the GLY group (6 vs. $10 \mathrm{mg} / \mathrm{day}$, (a)

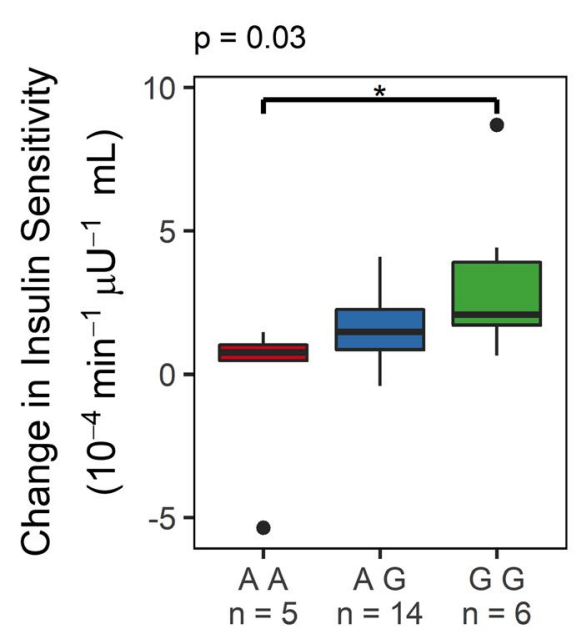

(c) OCT2 rs316019

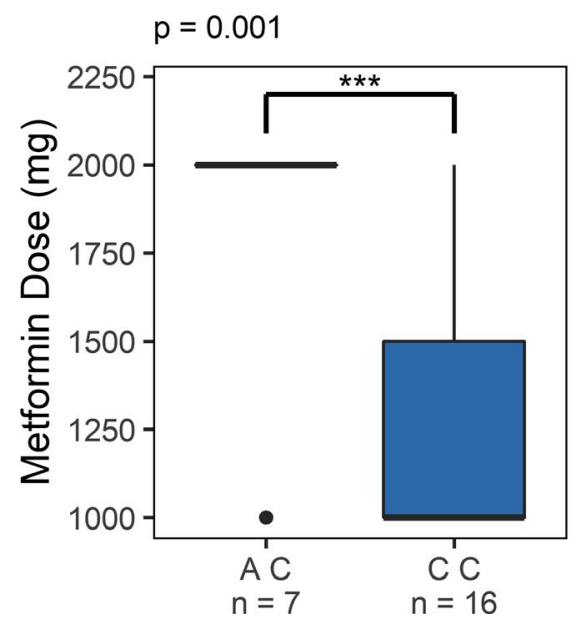

(b) PMAT rs2685753

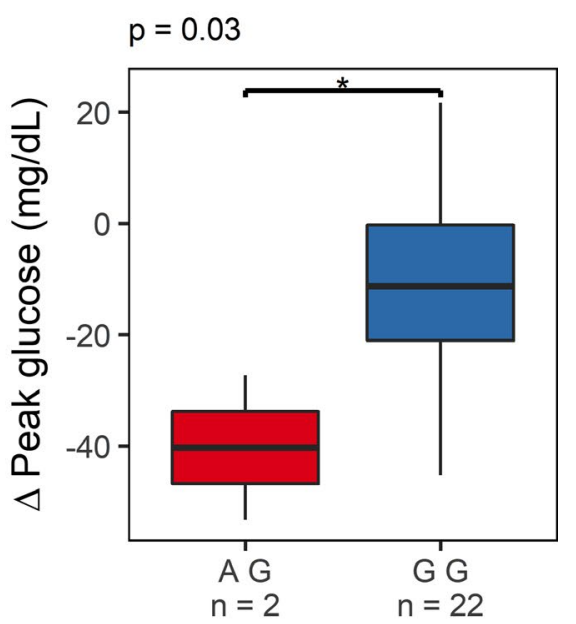

Figure 4 Effects of a splice variant of multidrug and toxin extrusion transporter 2 (MATE2-K) and organic cation transporter 2 (OCT2) genotype on metformin response and dose in women with gestational diabetes mellitus GDM who were adherent and completed the study. (a) Depicts a Tukey boxplot of the effects of MATE2-K genotype (rs12943590; AA, AG, and GG) on metformin (MET) pharmacologic activity (i.e., change in insulin sensitivity between study day 1 (SD1) and study day 2 (SD2), adjusted for gestational age-dependent changes) in women receiving MET monotherapy. (b) Depicts a Tukey boxplot of the effects of PMAT genotype (rs2685753; AG and GG) on change in peak glucose concentrations in women receiving MET monotherapy. (c) Depicts a Tukey boxplot of the effects of OCT2 genotype (rs316019, AC vs. CC) on MET dose on SD2 in women receiving MET monotherapy. $* P<0.05, * * P<0.01$, and $* * * P<0.001$. 
$P=0.005)$. Even so, average vector plots seem to show a small additional increase in $\beta$-cell responsivity in the $\mathrm{COMBO}$ group over the MET group, although this was not statistically significant (Figure 3). In the MET group, insulin concentrations were significantly lower with treatment than before treatment, reflecting MET's impact on SI and, thus, resulting in a lesser need for insulin and decreasing the demands on the pancreas.

Buchanan previously described the hyperbolic relationship between insulin secretion rate and SI in normal women and women with GDM in the third trimester of pregnancy and postpartum. Similar to our findings, he depicted the population with GDM to have a lower-than-normal DI. Interestingly, Buchanan depicted both pregnant and nonpregnant DI on the same curve. ${ }^{33}$ Subsequently, Buchanan's group published a 4-year longitudinal study describing declining DI in women with and without GDM, noting a faster decline in DI in individuals with GDM. ${ }^{34}$ In our study, "normal" pregnant women (i.e., those having normal values during their OGTT screening for GDM) had a 6\% decline in their DI over an average of 5.5 weeks between SD1 and SD2 (mean difference: $-200 \pm 400 \times 10^{-13}$ minute $\left.^{-2} \mu \mathrm{U}^{-1} \mathrm{~mL} ; P=0.04\right)$. This decreased ability to compensate for the degree of insulin resistance as pregnancy progresses, even among those who are "normal," aligns with the continuum of GDM described in the HAPO study and calls for careful consideration and standardization of diagnosis. ${ }^{32}$

Multiple bidirectional drug transporters are involved in the disposition of MET, including organic cation transporters 1-3 (OCT1, OCT2, and OCT3), as well as multidrug and toxin extrusion transporters 1, 2, and 2-K (MATE1, MATE2, and MATE2-K). OCT transporters and PMAT along with the MATE transporters work in series to move MET across the cell. ${ }^{35-37}$ MATE2-K (rs12943590 $\mathrm{G}>\mathrm{A}$ ) genetic variant is associated with increased promoter activity in vitro. ${ }^{38,39}$ In vivo, some but not all studies have reported that MATE2-K rs12943590 G>A genetic variant has been associated with increased MET renal clearance and decreased MET efficacy. We are the first to report the effect of MATE2-K on MET PD (i.e., the effect on SI following an MMTT). Consistent with reports of MATE2-K rs12943590 G>A genetic variant's impact on transporter activity, we found that women with GDM and MATE2-K AA genotype had a smaller increase in SI with MET than those with GG genotype (Figure 4a). ${ }^{40,41}$

In addition, women with GDM and OCT2 (rs316019) AC genotype required a higher MET dose than those with OCT2 CC genotype. Previously, it had been reported that the OCT2-808T genetic variant was associated with increased activity compared with the OCT2 reference in stably transfected HEK-293 cells in vitro. ${ }^{42}$ There are mixed reports on the effect of the OCT2 rs316019 A >C genetic variant on metformin disposition in vivo. ${ }^{43-47}$ It has been previously reported that individuals' heterozygous for the OCT2 variant allele (808G/T) had higher MET renal clearance than those homozygous for the OCT2 reference allele $(808 \mathrm{G} / \mathrm{G})$ in white volunteers. ${ }^{35}$ In contrast, two studies in Asian volunteers reported the opposite effect (i.e., those heterozygous for OCT2 variant allele $(808 \mathrm{G} / \mathrm{T}$ ) had lower MET renal clearance than those homozygous for OCT2 reference allele $(808 \mathrm{G} / \mathrm{G})){ }^{46,47}$ In this study, we did not find an association with OCT2 genotype and SI, but did find a relationship between OCT2 genotype and metformin dose. This is somewhat surprising given that during pregnancy we found no association with OCT2 genotype and metformin renal clearance. ${ }^{48}$ The explanation for this discrepancy is unclear. However, dosage is definitely a more confounded end point due to provider discretion than renal clearance or SI.

Last, there are conflicting results with respect to the effects of the PMAT (rs2685753 G>A) variant genotype and its association with MET exposure. ${ }^{49,50}$ Our results demonstrated that pregnant woman with GDM and the PMAT (rs2685753 G>A) AG genotype had a greater decrease in the MMTT peak glucose concentrations than those with GG genotype $(P=0.03)$. PMAT was not significantly associated with changes in metformin PD (change in SI) or metformin pharmacokinetics, ${ }^{48}$ which calls into question the mechanism by which PMAT genotype affected metformin's effect on MMTT peak glucose concentrations.

\section{Limitations}

With respect to the genetics portion of this study, the sample size is small to draw definitive conclusions with respect to the role of transporter genotypes in PD effects of MET. Larger studies are needed to explore the genetic associations with the biology of response.

In summary, this study, we believe for the first time, characterized the PD effects of GLY, MET, and COMBO treatment in women with GDM. Individual PD responses were variable, and none of the treatment approaches fully normalize the overall glycemic response. However, those that included MET shifted the overall DI closer to normal than those that primarily increased $\beta$-cell responsivity due to the hyperbolic shape of the DI curve and baseline parameters in the women with GDM. This suggests that maximizing MET and thereby SI first and then adding GLY to increase $\beta$-cell responsivity or adding insulin would be a more rational approach. Last, in the pregnant women with GDM, associations were found between MATE2-K genotype and MET's pharmacologic effect, OCT2, and MET dosage as well as PMAT and MET's effect on peak glucose concentrations.

\section{SUPPORTING INFORMATION}

Supplementary information accompanies this paper on the Clinical Pharmacology \& Therapeutics website (www.cpt-journal.com).

Figure S1. Changes in pharmacodynamic parameters with drug treatment of GDM. Histograms of the distribution of changes in total $\beta$-cell responsivity (Фtotal), SI, and DI for subjects receiving either COMBO (green), GLY (blue), or MET (purple) for the treatment of GDM adjusted for gestational age-dependent changes. Bar height indicates number of subjects. Zero, indicated with a vertical red line, represents no change. Figure S2. Glyburide monotherapy dosage titration. This figure depicts the study guide for glyburide monotherapy dosage titration. Starting dosage was glyburide $2.5 \mathrm{mg}$ orally twice daily and was titrated as high as $8.75 \mathrm{mg}$ orally three times daily. Provider's discretion was allowed. In addition, this figure depicts when SD2 MMTT should be completed. po, orally; bid, twice daily; tid, three time daily; PD, pharmacodynamic.

Figure S3. Metformin monotherapy dosage titration. This figure depicts the study guide for metformin monotherapy dosage titration. Starting dosage was metformin $500 \mathrm{mg}$ orally twice daily and was titrated as high as $2,000 \mathrm{mg} /$ day in divided doses. Provider's discretion was allowed. In addition, this figure depicts when study day 2 MMTT should be completed. po, orally; bid, twice daily; tid, three time daily; qid, four times daily; PD, pharmacodynamic.

Figure S4. Glyburide and metformin combination therapy dosage titration. This figure depicts the study guide for glyburide and metformin dosage titration in the combination therapy group. Starting glyburide dosage was $2.5 \mathrm{mg}$ orally twice daily and titrated as high as $10 \mathrm{mg}$ 
orally twice daily. Starting metformin dosage was $500 \mathrm{mg}$ orally twice daily and titrated as high as $2,000 \mathrm{mg} /$ day in divided doses. Provider's discretion was allowed. In addition, this figure depicts when study day 2 MMTT should be completed. po, orally; bid, twice daily; tid, three time daily; qid, four times daily; PD, pharmacodynamic.

Table S1. Demographics of all eligible subjects with gestational diabetes and gestational age-matched healthy pregnant subjects.

\section{ACKNOWLEDGMENTS}

In memoriam, we acknowledge the tremendous contribution of David A. Flockhart, PhD, MD, to the Obstetric-fetal Pharmacology Research Unit Network. We also thank the research coordinators and nurses, including Alisha Bouge, Claudine Hernandez, Karen Hays, Ira Kantrowitz-Gordon, Anna Lemchen, Heather Follen, Holly West, Julie Croxford, Dawn Fisher, Wenchen Zhao, Becky Cypher, and Janie Klank for their hard work in completing this study.

\section{FUNDING}

This research was supported in part by the Eunice Kennedy Shriver National Institute of Child Health and Human Development grants \#U10HD063094, U10HD047892, U10HD097905, U10HD047891, U10HD057753, and the National Institutes of Health (NIH) National Center for Advancing Translational Science through the Clinical and Translational Science Awards Program grants \#ULITR000423, TLITR000422, ULITR001108, National Institute of General Medical Sciences R01GM124264, and unrestricted research funds from the University of Washington. The content of this manuscript is solely the responsibility of the authors and does not necessarily represent the official views of the Eunice Kennedy Shriver National Institute of Child Health and Human Development or the National Institute of Health. In addition, the views expressed are those of the author(s) and do not reflect the official policy or position of the US Army Medical Department, Department of the Army, Department of Defense, or the US Government. The investigators have adhered to the policies for protection of human subjects as prescribed in 45 CFR 46 .

\section{CONFLICT OF INTEREST}

The authors declare that, at the time of the conduct and analysis of the study, they had no competing interests for this work. However, since the completion of the study, D.L.S.'s affiliation has changed to PRA Health Sciences.

\section{AUTHOR CONTRIBUTIONS}

D.L.S., L.M.S., X.M., D.D.S., S.K.F.-N., M.A., Sh.C., St.C., R.V., D.M.H., S.K.Q., L.S.H., A.T.T., T.A.M., K.E.T., L.M.B., Z.R., Z.B., T.R.E., and M.F.H. wrote the manuscript. X.M., D.D.S., K.E.T., Z.R., Z.B., L.M.B., T.R.E., and M.F.H. designed the research. S.K.F.-N., Sh.C., St.C., D.M.H., A.T.T., T.A.M., L.M.B., T.R.E., and M.F.H. performed the research. D.L.S., L.M.S., X.M., D.D.S., and M.F.H. analyzed the data. R.V. and S.K.Q. contributed new reagents/analytical tools.

1. Jovanovic, L. \& Pettitt, D.J. Gestational diabetes mellitus. JAMA 286, 2516-2518 (2001).

2. Paglia, M.J. \& Coustan, D.R. Gestational diabetes: evolving diagnostic criteria. Curr. Opin. Obstet. Gynecol. 23, 72-75 (2011).

3. HAPO Study Cooperative Research Group. The Hyperglycemia and Adverse Pregnancy Outcome (HAPO) Study. Int. J. Gynaecol. Obstet. 78, 69-77 (2002).

4. Committee on Practice Bulletins-Obstetrics. ACOG Practice Bulletin No. 190: gestational diabetes mellitus. Obstet. Gynecol. 131, e49-e64 (2018).

5. Committee on Practice, Bulletins-Obstetrics. Practice Bulletin No. 137: gestational diabetes mellitus. Obstet. Gynecol. 122, 406-416 (2013).

6. Langer, O., Conway, D.L., Berkus, M.D., Xenakis, E.M. \& Gonzales, O.A. Comparison of glyburide and insulin in women with gestational diabetes mellitus. N. Engl. J. Med. 343, 1134-1138 (2000).

7. Camelo Castillo, W., Boggess, K., Sturmer, T., Brookhart, M.A., Benjamin, D.K. Jr \& Jonsson Funk, M. Trends in glyburide compared with insulin use for gestational diabetes treatment in the United States, 2000-2011. Obstet. Gynecol. 123, 11771184 (2014).

8. Feldman, J.M. Glyburide: a second-generation sulfonylurea hypoglycemic agent. History, chemistry, metabolism, pharmacokinetics, clinical use and adverse effects. Pharmacotherapy 5, 43-62 (1985).

9. Kirpichnikov, D., McFarlane, S.I. \& Sowers, J.R. Metformin: an update. Ann. Intern. Med. 137, 25-33 (2002).

10. Liang, H.-L., Ma, S.-J., Xiao, Y.-N. \& Tan, H.-Z.. Comparative efficacy and safety of oral antidiabetic drugs and insulin in treating gestational diabetes mellitus. An updated PRISMAcompliant network meta-analysis. Medicine (Baltimore) 96, e7939 (2017).

11. Hebert, M.F. et al. Are we optimizing gestational diabetes treatment with glyburide? The pharmacologic basis for better clinical practice. Clin. Pharmacol. Ther. 85, 607-614 (2009).

12. Dalla Man, C., Caumo, A. \& Cobelli, C. The oral glucose minimal model: estimation of insulin sensitivity from a meal test. IEEE Trans. Biomed. Eng. 49, 419-429 (2002).

13. Breda, E., Cavaghan, M.K., Toffolo, G., Polonsky, K.S. \& Cobelli, C. Oral glucose tolerance test minimal model indexes of betacell function and insulin sensitivity. Diabetes 50, 150-158 (2001).

14. Bergman, R.N., Phillips, L.S. \& Cobelli, C. Physiologic evaluation of factors controlling glucose tolerance in man: measurement of insulin sensitivity and beta-cell glucose sensitivity from the response to intravenous glucose. J. Clin. Invest. 68, 1456-1467 (1981).

15. Cobelli, C. et al. Assessment of beta-cell function in humans, simultaneously with insulin sensitivity and hepatic extraction, from intravenous and oral glucose tests. Am. J. Phys. Endocrin. Metab. 293, E1-E15 (2007).

16. Bergman, R.N. Minimal model: perspective from 2005. Hormone Res. 64 (suppl. 3), 8-15 (2005).

17. Jacobson, G.F., Ramos, G.A., Ching, J.Y., Kirby, R.S., Ferrara, A. \& Field, D.R. Comparison of glyburide and insulin for the management of gestational diabetes in a large managed care organization. Am. J. Obstet. Gynecol. 193, 118-124 (2005).

18. Kremer, C.J. \& Duff, P. Glyburide for the treatment of gestational diabetes. Am. J. Obstet. Gynecol. 190, 1438-1439 (2004).

19. Groop, L. et al. Comparison of pharmacokinetics, metabolic effects and mechanisms of action of glyburide and glipizide during long-term treatment. Diabetes Care 10, 671-678 (1987).

20. Chmait, R., Dinise, T. \& Moore, T. Prospective observational study to establish predictors of glyburide success in women with gestational diabetes mellitus. J. Perinatol. 24, 617-622 (2004).

21. Rowan, J.A., Hague, W.M., Gao, W., Battin, M.R., Moore, M.P. \& Mi, G.T.I. Metformin versus insulin for the treatment of gestational diabetes. N. Engl. J. Med. 358, 2003-2015 (2008).

22. Moore, L.E., Clokey, D., Rappaport, V.J. \& Curet, L.B. Metformin compared with glyburide in gestational diabetes: a randomized controlled trial. Obstet. Gynecol. 115, 55-59 (2010).

23. Bandi, Z.L., Fuller, J.B., Bee, D.E. \& James, G.P. Extended clinical trial and evaluation of glucose determination with the Eastman Kodak Ektachem GLU/BUN Analyzer. Clin. Chem. 27, 27-34 (1981).

24. Haffner, S.M., Mykkanen, L., Stern, M.P., Valdez, R.A., Heisserman, J.A. \& Bowsher, R.R. Relationship of proinsulin and insulin to cardiovascular risk factors in nondiabetic subjects. Diabetes 42, 1297-1302 (1993).

25. Wiedmeyer, H.M. et al. International comparison of C-peptide measurements. Clin. Chem. 53, 784-787 (2007).

26. Caumo, A., Bergman, R.N. \& Cobelli, C. Insulin sensitivity from meal tolerance tests in normal subjects: a minimal model index. J. Clin. Endocrinol. Metab. 85, 4396-4402 (2000).

27. Dalla Man, C., Caumo, A., Basu, R., Rizza, R., Toffolo, G. \& Cobelli, C. Minimal model estimation of glucose absorption and 
insulin sensitivity from oral test: validation with a tracer method. Am. J. Physiol. Endocrinol. Metab. 287, E637-E643 (2004).

28. Dalla Man, C. et al. Two-hour seven-sample oral glucose tolerance test and meal protocol: minimal model assessment of beta-cell responsivity and insulin sensitivity in nondiabetic individuals. Diabetes 54, 3265-3273 (2005).

29. R Core Team. R: A Language and Environment for Statistical Computing (R Foundation for Statistical Computing, Vienna, Austria, 2018) <https://www.R-project.org/>.

30. Wickham, H. ggplot2: Elegant Graphics for Data Analysis (SpringerVerlag, New York, NY, 2016).

31. Eyal, S. et al. Pharmacokinetics of metformin during pregnancy. Drug Metab. Dispos. 38, 833-840 (2010).

32. The HAPO Study Cooperative Research Group et al. Hyperglycemia and adverse pregnancy outcomes. N. Engl. J. Med. 358, 19912002 (2008).

33. Buchanan, T.A. Pancreatic B-cell defects in gestational diabetes: implications for the pathogenesis and prevention of type 2 diabetes. J. Clin. Endocrinol. Metab. 86, 989-993 (2001).

34. Xiang, A.H. et al. Longitudinal changes in insulin sensitivity and beta cell function between women with and without a history of gestational diabetes mellitus. Diabetologia 56, 2753-2760 (2013).

35. Nies, A.T., Damme, K., Kruck, S., Schaeffeler, E. \& Schwab, M. Structure and function of multidrug and toxin extrusion proteins (MATEs) and their relevance to drug therapy and personalized medicine. Arch. Toxicol. 90, 1555-1584 (2016).

36. Motohashi, H. et al. Precise comparison of protein localization among OCT, OAT and MATE in human kidney. J. Pharm. Sci. 102, 3302-3308 (2013).

37. Motohashi, H. \& Inui, K.-I. Organic cation transporter OCTs (SLC22) and MATEs (SLC47) in the human kidney. AAPS J. 15, 581-588 (2013).

38. Choi, J.H. et al. A common 5'-UTR variant in MATE2-K is associated with poor response to metformin. Clin. Pharmacol. Ther. 90, 674-684 (2011).

39. Yoon, H., Cho, H.Y., Yoo, H.D., Kim, S.M. \& Lee, Y.B. Influence of organic cation transporter polymorphisms on the population pharmacokinetics of metformin in healthy subjects. AAPS J. $\mathbf{1 5}$, 571-580 (2013).

40. Chung, J.-Y. et al. Functional characterization of MATE2-K genetic variants and their effects on metformin pharmacokinetics. Pharmacogenet. Genomics 23, 365-373 (2013).

41. Stocker, S.L. et al. The effect of novel promoter varints in MATE1 and MATE2 on the pharmacokinetics and pharmacodynamics of metformin. Clin. Pharmacol. Ther. 93, 186-194 (2013).

42. Chen, Y., Zhang, S., Sorani, M. \& Giacomini, K.M. Transport of paraquat by human organic cation transporters and multidrug and toxic compound extrusion family. J. Pharmacol. Exp. Ther. 322, 695-700 (2007).

43. Christensen, M.M.H. et al. A gene-gene interaction between polymorphisms in OCT2 and MATE1 genes influences the renal clearance of metformin. Pharmacogenet. Genom. 23, 526-534 (2013).

44. Tkac, I. et al. Pharmacogenomic association between a variant in SLC47A1 gene and therapeutic response to metformin in type 2 diabetes. Diabetes Obesity Metab. 15, 189-191 (2013).

45. Chen, Y. et al. Effect of genetic variation in organic cation transporter 2 on the renal elimination of metformin. Pharmacogenet. Genomics 19, 497-504 (2009).

46. Wang, Z.-J., Yin, O.Q.P., Tomlinson, B. \& Chow, M.S.S. OCT2 polymorphisms and in vivo renal functional consequence: studies with metformin and cimetidine. Pharmacogenet. Genomics 18, 637-645 (2008).

47. Song, I.S. et al. Genetic variants of the organic cation transporter 2 influence the disposition of metformin. Clin. Pharmacol. Ther. 84, 559-562 (2008).

48. Liao, M.Z. et al. Effects of pregnancy on the pharmacokinetics of metformin. Drug. Metab. Dispos. (2020). In Press.

49. Moon, S.J., Oh, J., Lee, S.H., Choi, Y., Yu, K.-S. \& Chung, J.-Y. Effect of plasma membrane monoamine transporter genetic variants on pharmacokinetics of metformin in humans. Transl. Clin. Pharmacol. 26, 79-85 (2018).

50. Staiger, H., Schaeffeler, E., Schwab, M. \& Haring, H.-U. Pharmacogenetics: implications for modern type 2 diabetes therapy. Rev. Diabet. Stud. 12, 363-376 (2015). 